\title{
Role of lung ultrasound in differentiating lung congestion and lung infection in paediatric cardiac patients
}

\author{
Mahmoud Kamal Moustafa, Maha Elkholy, Gamal Askr, Duaa Mohammed Rafaat, Amal El-Sisi
}

\begin{abstract}
Moustafa MK, El-Kholy M, Askr G, et al. Role of lung ultrasound in differentiating lung congestion and lung infection in paediatric cardiac patients. Curr Res Cardiol 2017;4(3):36-39.
\end{abstract}

INTRODUCTION: Lung sonography in the emergency situations became one of the most appropriate studies. This technique allows us to diagnose major causes of acute respiratory distress at the bedside without major interventions. It is easy, costless approach to diagnose many lung diseases. The success of this technique depends on its simplicity to discover the sonographic artifacts which indicate certain lung pathology. The A-profile associates anterior lung sliding with $\mathrm{A}$ lines. A lines are horizontal repetition artifacts of the pleural line. The B-profile associates anterior lung sliding with B lines. B lines appear as shining vertical lines arising from the pleural line and reach the edge of the screen.

PATIENTS AND METHODS: A descriptive study included cardiac patients admitted to the pediatric cardiology ward for six months. They had their data completely revised and their treatment recorded and diagnosis traced with a direct question: is it congestion or infection. A lung ultrasound was done

Ongenital heart disease is a structural malformation of the heart or great blood vessels which happens during intrauterine life $(1,2)$. Pulmonary congestion is a major cause of morbidity and mortality in heart failure (3). Incomplete treatment of congestion during admission is associated with increased morbidity and mortality, whereas patients discharged free from congestion show better prognosis (4). Lung ultrasound (LUS) is a simple approach to confirm pulmonary congestion, by evaluation of B-lines (5). B-lines are the sonographic sign of the pulmonary congestion, reflecting the pulmonary interstitial oedema in patients with acute heart failure. Studies have shown their use in diagnosis of pulmonary causes of acute dyspnea (6).

The aim of the study is

- To describe how far lung congestion or infection is behind our cardiac patients thus, lung ultrasound will enable us to use antibiotics or anti-failure measures (Diuretics such as Furosemide and Spironolactone plus Angiotensin-converting enzyme Inhibitors and Angiotensin II Receptor Blockers plus Digitalis Glycosides) when needed.

- To evaluate the diagnostic accuracy of bedside lung ultrasound in pediatric cardiac patients admitted to the pediatric cardiology unit with suspected pneumonia or heart failure or both and final diagnosis at discharge.

- How much lung sonar could change our decision and help us for an easier costless diagnosis.

Subjects

Cardiac patients one month to sixteen years of age admitted to the pediatric cardiology unit (From first of November 2016 to end of April 2017).

Inclusion criteria

Cardiac patients one month to sixteen years of age admitted to the pediatric cardiology unit with respiratory infection or manifestations of heart failure whatever the cardiac anomaly. to try to assure the diagnosis. Also, chest $\mathrm{x}$ ray and echo correlation were assessed.

RESULTS: Sixty patients were enrolled over a period of six months $(58 \%$ male, with the mean age \pm SD was $(17.33 \pm 30.91)$ months. Twenty-seven of the sixty patients had a B-profile, thirty-five had A-profile, A\&B profile was found in six cases, hypoechoic areas were found in nine cases. Lung ultrasound appeared to be normal in three cases.

CONCLUSION: By applying use of lung ultrasound in pediatric cardiology ward to diagnose or to confirm the diagnosis of chest infection, air trapping, lung congestion up to pulmonary edema, thus differentiating which line of treatment is better to the patients, antibiotics or bronchodilators or antifailure medication avoiding blind use of antibiotics.

Key Words: Lung ultrasound; Congenital heart disease; Pulmonary edema; Heart failure; Pulmonary congestion; B-profile; A-profile

Abbreviations: CHD: Congenital Heart Disease; LUS: Lung Ultrasound; JVP: Jugular Venous Pressure

\section{Exclusion criteria}

-Age less than one month

- If admission is due to cyanotic spells or central nervous system cause.

Tools of study

A descriptive study based on complete history and physical examination of the patients. Transthoracic LUS examinations were performed with available ultrasound machines, equipped with a linear probe with frequencies ranging from 6 to $9 \mathrm{MHz}$. The sonographer performed a complete scanning of the chest in all children. LUS examination consisted of both longitudinal and transversal sections. Correlation with chest x-ray, echocardiography, and complete blood count was done

\section{RESULTS}

The study was conducted on children admitted to the pediatric cardiology ward in Assuit University Children Hospital over 6 months. The study included 60 cases.

A-Demographic data (Figures 1-3)

B- History and presenting symptoms in studied patients (Figure 4)

C-Clinical findings of studied cases (Figure 5)

D-Laboratory and radiological findings among studied case (Figures $6-10)$.

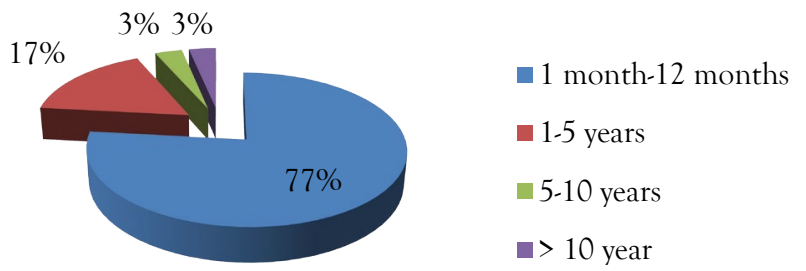

Figure 1) The age distribution of the studied cases

Pediatrics department, Faculty of Medicine, Assiut University, Egyp

Correspondence: Dr Mahmoud Kamal Moustafa, Pediatrics department, Faculty of Medicine, Assiut University, Egypt, Telephone+201009983881, e-mail hotta_forever@ yahoo.com

Received: July 05, 2017, Accepted: August 14, 2017, Published: August 16, 2017

This open-access article is distributed under the terms of the Creative Commons Attribution Non-Commercial License (CC BY-NC) (http:// creativecommons.org/licenses/by-nc/4.0/), which permits reuse, distribution and reproduction of the article, provided that the original work is properly cited and the reuse is restricted to noncommercial purposes. For commercial reuse, contact reprints@pulsus.com
} 
$42 \%$

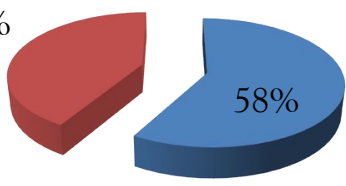

Figure 2) The sex distribution of the studied cases

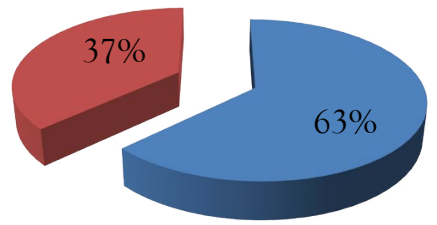

- Rural

- Urban

Figure 3) The residence distribution of the studied cases

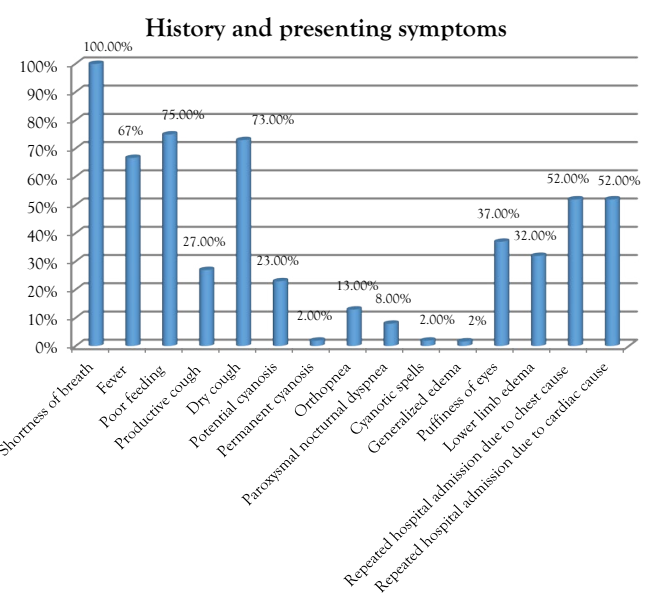

Figure 4) History and presenting symptoms in studied patients

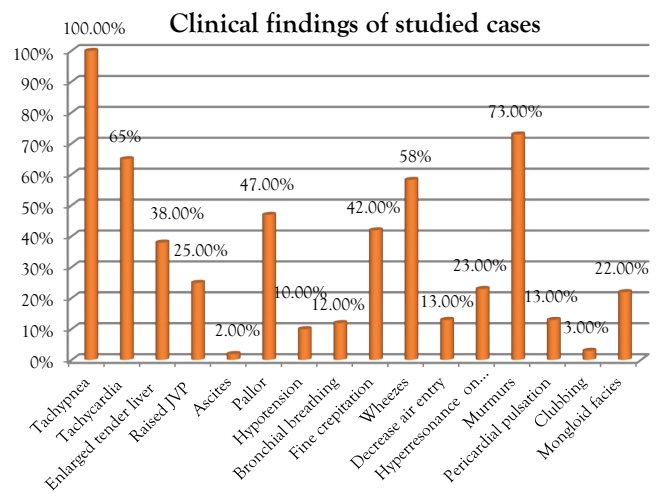

Figure 5) Clinical findings of studied cases

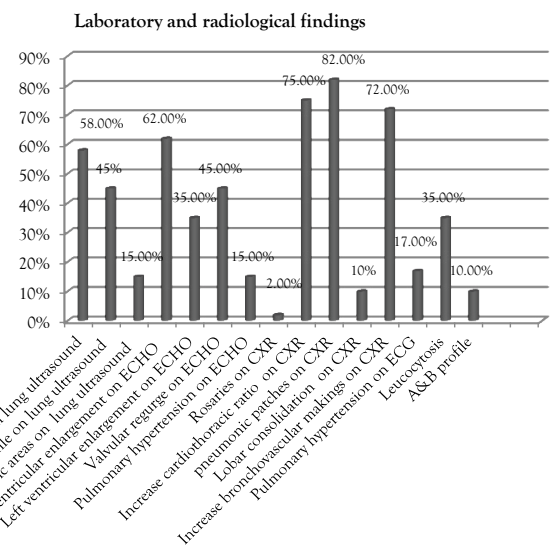

Figure 6) Laboratory and radiological findings

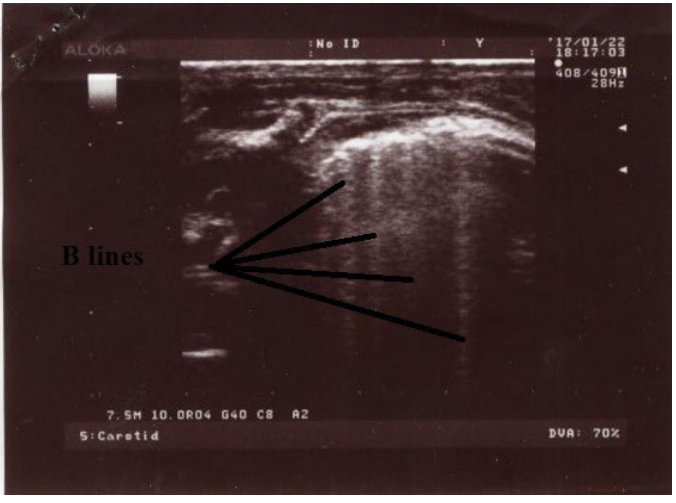

Figure 7) Multiple B lines (black lines) vertical hyperechoic artifacts that arise from the pleural line to the end of the screen

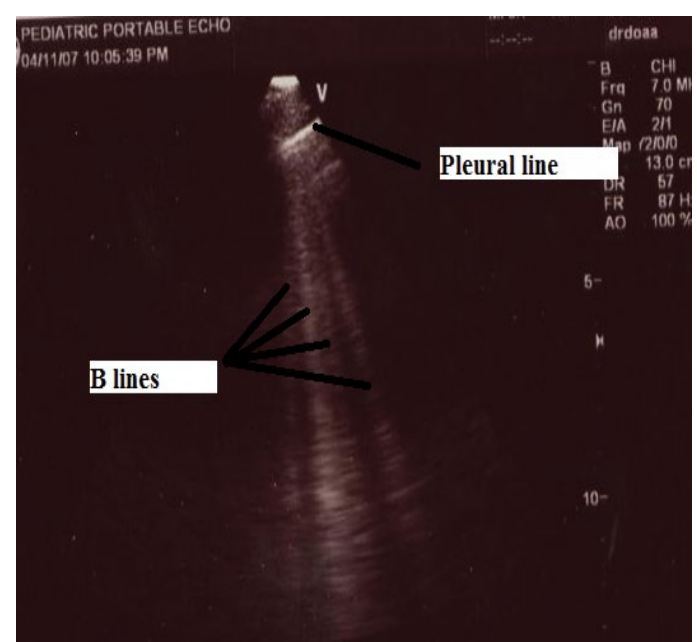

Figure 8) Multiple B lines Ëpleural line which appeared hyper echoic elements

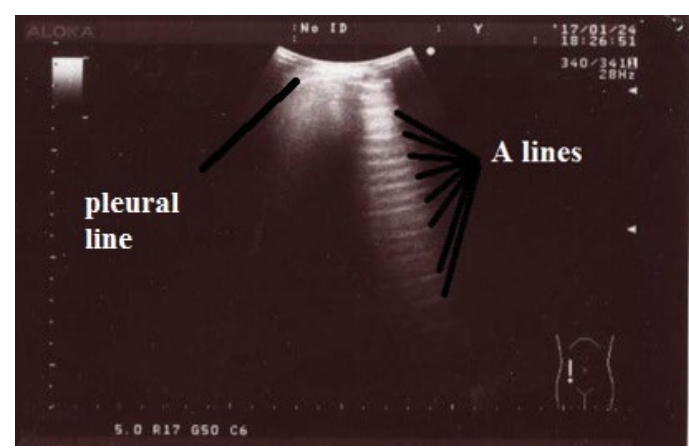

Figure 9) Multiple A lines horizontal artifacts parallel to the pleural line

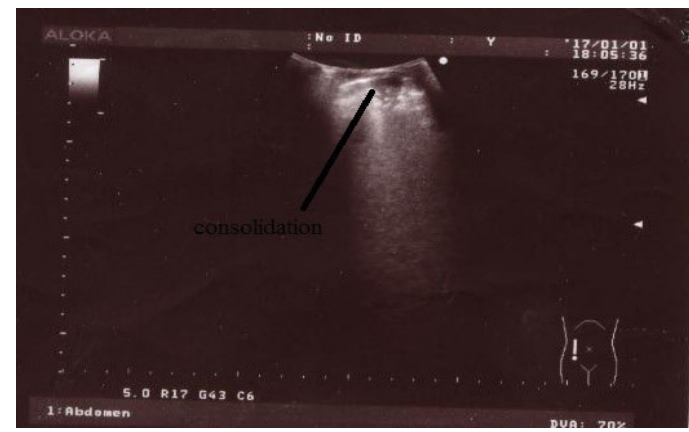

Figure 10) Lung consolidation: hypoechoic area with internal hyperechoic elements, representing air bronchograms

\section{DISCUSSION}

This is a descriptive study to discuss the role of lung ultrasound in differentiating lung congestion and lung infection in pediatric cardiac patients.

Curr Res Cardiol Vol 4 No 3 Autumn 2017 
This study was done on cardiac patients admitted to the pediatric cardiology ward at Assiut University Children \s Hospital for 6 months from first of November 2016 to end of April 2017 from age of 1 month to 16 years of age with respiratory infection or manifestations of heart failure whatever the cardiac anomaly. That was the study only inclusion criteria.

Exclusion criteria were if the admission was due to cyanotic spells or central nervous system cause and neonates.

This study included 60 cases, 35 males (58\%) and 25 females (42\%). Caiulo et al. who started a similar study also had 53 males (52\%) and 49 females (48\%).

According to the age in this study, the majority of cases were in the infancy time by $77 \%$ followed by the age range $1-5$ years $17 \%$, lastly $5-10$ years and > 10 years were $3 \%$ (mean 17.33 months, $\mathrm{SD} \pm 30.91$ ).

However, Iorio et al. mentioned that ages ranged from 2 months to 12.5 years (mean $3.5 \mathrm{y}$, standard deviation \pm 3.1 ) .

According to the history and presenting symptoms in this study, it was found that all cases whatever their diagnosis, their main complaint was shortness of breath. This was followed by two symptoms nearly on the same foot of equality; poor feeding $75 \%$ \& dry cough $73 \%$. Then comes fever $67 \%$.

According to the general examination in the present study, it was found that the main clinical finding was tachypnea in every case (100\%).

Signs of heart failure in this study as tachycardia $(65 \%)$, enlarged tender liver $(38 \%)$, puffiness of eyes (37\%), lower limb edema (32\%) and raised JVP (25\%).

The results are in agreement with N. Jayaprasad who reported that the clinical features suggestive of heart failure in infants include tachypnea, feeding difficulty and diaphoresis. Irritability with feeding, sweating, and even refusal of feeds was also common.

Also D. Masarone et al. study which reported that the typical presentation of heart failure in infants and young children is difficulty in feeding, while in older children and adolescence: fatigue, shortness of breath, tachypnea, and exercise intolerance were the main symptoms. Abdominal pain, oliguria, and leg pitting edema could be present.

Signs of chest infection in this study were wheezes (58\%) mainly then comes fine crepitation (42\%) followed by bronchial breathing (12\%) and decrease air entry (13\%).

This in coincidence with Qiang Qin and Kun-ling Shen as they mentioned that examination findings in suspected cases of pneumonia include crepitations decreased breath sounds, bronchial breathing (findings indicating consolidated lung parenchyma) and wheezing (more common in pneumonia caused by atypical bacteria and viruses).

Actually, Assiut University Children"s Hospital did not offer the facility to detect which type of pneumonia according to the organism but much expected that wheezes were of viral origin.

Also, two symptoms were found to be equal in our studied cases, repeated hospital admission due to chest cause\& repeated hospital admission due to cardiac cause $52 \%$ each.

F. Healy et al. study which reported that respiratory tract infection in children with CHD is an important cause of morbidity and mortality including respiratory failure, prolonged mechanical ventilation, and hospitalization. These patients often have many contributing factors that place them at increased risk for respiratory tract infection including malnutrition, aspiration, prolonged duration of tracheal intubation and/or mechanical ventilation and previous use of extended spectrum antibiotics that inhibit intestinal flora. This confirms the main focus of the present study is to differentiate between heart failure and chest infection.

Pallor was found in $47 \%$ of our studied cases which agree with D. Masarone et al. as it reported that complete blood count is useful to assess anemia, which may cause or aggravate heart failure.

LUS Findings in this study showed that A profile was found in 35 cases $(58 \%)$, however in Bitar et al, A profile was found in 14 patients of total 61 patients (23\%).

B profile was found in 27 cases (45\%), this agrees with Caiulo et al. which showed B lines in 59 patients (55\%).
However, in Bitar et al. B profile was found in 47 patients of total 61 patients (77\%).

E. Platz et al. reported that B lines were found in 32\% of studied cases.

Hypoechoic areas were found in 9 cases (15\%) in this study; however, Caiulo et al. showed lung consolidations in 65 cases (78\%). A\&B profile was found in 6 cases $(10 \%)$.

LUS appeared to be normal in 3 cases $(5 \%)$, this agree with Caiulo et al. which showed that LUS appeared to be normal in $1 / 89$ children with pneumonia.

CXR findings in the current study showed that pneumonic patches were found in 49 cases $(82 \%)$, increase cardiothoracic ratio in 45 cases $(75 \%)$, increase bronchovascular makings in 43 cases (72\%), lobar consolidation in 6 cases $(10 \%)$.

In this study, three patients with negative consolidation on chest $\mathrm{x}$ ray were positive with hypoechoic areas in LUS.

However; Iorio et al. showed that chest radiography detected pneumonia in 25 patients $(86.2 \%)$.

According to radiological findings of echocardiography in this study; decreased fractional shortening below $28 \%$ was found in 29 cases. By examination of these cases it was found that all of them had tachypnea and tachycardia, 19 cases had enlarged tender liver, 15 cases had raised JVP and when LUS was done to these patients; B lines were found in 27 cases. Left ventricular enlargement in 21 cases (35\%), valvular regurge in 27 cases (45\%), and pulmonary hypertension in 9 cases $(15 \%)$.

The study results are in agreement with Kirkpatrick et al. which reported that heart failure is classically described as left ventricular (LV) dysfunction leading to congestion and reduced systemic perfusion, most often manifesting symptomatically as dyspnea and fatigue.

Leukocytosis was found in 21 cases (35\%) in this study which agree with D. Masarone et al. which mentioned that leukocytosis may result from stress or signal to an underlying infection.

According to the management in this study, baseline antibiotics were prescribed in all cases which is the usual practice in the unit. It is a pity to do this but it was a must because bacterial infection is endemic in the locality \& to protect against hospital acquired infection. All cases received Ampicillin/ sulbactam. The decision was taken to upgrade the antibiotics in 30\% of cases in the form of ceftazidime in 5 cases, cefotaxime in 10 cases and cefepime in 3 cases. 50 cases $(83 \%)$ were on anti-failure medication. 36 cases were already on anti-failure medication before admission. Anti-failure medications were newly prescribed in 14 cases. In this study, 55 cases (92\%) clinically improved and were discharged, while 5 cases $(8 \%)$ died as they were suffering from severe respiratory distress. Three cases died due to fulminant chest infection and two cases died due to heart failure and cardiogenic shock. The duration of admission ranged from 3 days up to 11 days.

On congruence with C.C. Engelings et al. study which reported that the leading causes of death in congenital heart diseases were heart failure and sudden cardiac death.

Regarding the previous results, to our knowledge; this is a unique study to use combination between history, physical examination and investigations as lung ultrasound, echocardiography, complete blood count and chest $\mathrm{x}$ ray. This study has several strengths and unique work. 60 children aged from 1 month to 16 years were enrolled and performed all tests in a busy tertiary hospital, suggesting that chest sonar can be a useful approach. Also in this work, there were two experts; one in echocardiography and the other in lung ultrasound. It is believed that lung ultrasound could diagnose by itself pulmonary edema, pneumonia, air trapping even pleural effusion and pneumothorax without use of other modalities such as chest X ray and CT chest. The main limitation of radiography is the risk of damage from ionizing radiation.

Blind use of antibiotics should be avoided as it could be cause of drug resistance and repeated chest infections in cardiac patients as it was mentioned before.

\section{CONCLUSION}

Moreover by applying use of lung ultrasound in pediatric cardiology ward to diagnose or to confirm the diagnosis of chest infection, air trapping, lung congestion up to pulmonary edema. Differentiating which line of treatment is better to the patients, antibiotics or bronchodilators or anti-failure 
medication avoiding blind use of antibiotics. Hope to apply LUS as day and night approach for progress on anti-failure medication and help changing line of treatment together with other tools as biomarkers and inferior vena cava index (7).

\section{REFERENCES}

1. Volpicelli G, Cardinale L, Garofalo G, et al. Usefulness of lung ultrasound in the bedside distinction between pulmonary edema and exacerbation of COPD. Emerg Radiol 2008;15:145-51.

2. Bhalla S, Javidan-Nejad C, Bierhals AJ, et al. CT in the evaluation of congenital heart disease in children, adolescents, and young adults. Curr Treat Options Cardiovasc Med 2008;10:425-32.

3. Gheorghiade M, Follath F, Ponikowski P, et al. European Society of Cardiology; European Society of Intensive Care Medicine. Assessing and grading congestion in acute heart failure: A scientific statement from the acute heart failure committee of the heart failure association of the European Society of Cardiology and endorsed by the European Society of Intensive Care Medicine. Eur J Heart Fail. 2010;12:423-33.
4. Gattis WA, O'Connor CM, Gallup DS, et al. Pre-discharge initiation of carvedilol in patients hospitalized for decompensated heart failure: Results of the initiation management pre-discharge: process for assessment of carvedilol therapy in heart failure (IMPACT-HF) trial. J Am Coll Cardiol. 2004;43:1534-41.

5. Volpicelli G, Elbarbary M, Blaivas $\mathrm{M}$, et al. International Liaison Committee on Lung Ultrasound (ILC-LUS) for International Consensus Conference on Lung Ultrasound (ICC-LUS). International evidencebased recommendations for point-of-care lung ultrasound. Intensive Care Med. 2012;38:577-91.

6. Pivetta E, Goffi A, Lupia E, et al. Lung ultrasound implemented diagnosis of acute decompensated heart failure in the emergency department - a simeu multicenter study. Chest 2015;148(1):202-10.

7. Blehar D, Dickman E, Gaspari R. Identification of congestive heart failure via respiratory variation of inferior vena cava diameter. Am J Emerg Med 2009;27:71-5. 